Revue de droit comparé du travail et de la sécurité sociale

3 | 2019

Les migrations internationales de travail

\title{
Les principales lois adoptées lors de la session ordinaire 2018-2019 de la Diète
}

Masahiko Iwamura

\section{(2) OpenEdition}

1 Journals

Édition électronique

URL : https://journals.openedition.org/rdctss/1515

DOI : 10.4000/rdctss. 1515

ISSN : 2262-9815

Éditeur

Centre de droit comparé du travail et de la sécurité sociale

Édition imprimée

Date de publication : 1 novembre 2019

Pagination : 184-187

ISSN : 2117-4350

\section{Référence électronique}

Masahiko Iwamura, « Les principales lois adoptées lors de la session ordinaire 2018-2019 de la

Diète ", Revue de droit comparé du travail et de la sécurité sociale [En ligne], 3 | 2019, mis en ligne le 01 novembre 2021, consulté le 12 novembre 2021. URL : http://journals.openedition.org/rdctss/1515 ; DOI : https://doi.org/10.4000/rdctss. 1515

\section{(c) $(7)(8$}

Revue de droit comparé du travail et de la sécurité sociale est mise à disposition selon les termes de la Licence Creative Commons Attribution - Pas d'Utilisation Commerciale - Pas de Modification 4.0 International. 


\section{MASAHIKO IWAMURA}

UNIVERSITÉ DE TOKYO, Président de la Commission Centrale des Relations de Travail

\section{LES PRINCIPALES LOIS ADOPTÉES LORS DE LA SESSION ORDINAIRE 2018-2019 DE LA DIÈTE}

Dans la perspective de la campagne électorale pour les élections législatives de «Sangi-in» (2 ${ }^{\text {ème }}$ Chambre de la Diète) - qui se sont déroulées en juillet 2019 -, ni le Gouvernement du Premier Ministre Shinzo ABE, ni les partis au pouvoir (parti libéral-démocrate et parti Komei soutenu par une secte de bouddhistes « Soka Gakkai »), n'ont souhaité engager des débats potentiellement houleux à la Diète avec les partis d'oppositions sur le thème « brûlant » de la politique sociale au Japon. De ce fait, le ministère de la santé, du travail et des affaires sociales (MSTA) a déposé seulement 5 projets de loi au cours de la $198^{\mathrm{e}}$ session de la Diète. Finalement adoptés, ces projets de loi apportent pourtant quelques réformes intéressantes ici examinées.

\section{I - LA LUTTE CONTRE LE HARCÈLEMENT SUR LE LIEU DE TRAVAIL}

Parmi ces réformes, le renforcement de la politique de lutte contre le harcèlement sur le lieu de travail mérite une attention particulière. Pour la première fois dans la législation du travail au Japon, le harcèlement lié à des relations de pouvoir est défini par la loi.

En effet, la loi n 24 du 5 mai 2019 a inséré dans la « Loi sur la promotion de l'ensemble des politiques de travail ainsi que sur la stabilité de l'emploi des salariés et l'amélioration de leur vie professionnelle » (loi n 132 du 21 juillet 1966) un nouvel article qui définit le "harcèlement lié à des relations de pouvoir» comme "les comportements et les paroles d'un supérieur contre un ou des subordonnés sur le lieu de travail, dans un contexte hiérarchique, qui ne peuvent être justifiés ni par la nécessité de management, ni par sa pertinence, et dégradent ainsi l'environnement de travail ».

Dès le $1^{\text {er }}$ avril 2020, l'employeur sera donc tenu d'instaurer un ou des dispositifs permettant de prendre toutes mesures appropriées ou relatives à la gestion des ressources humaines pour répondre à une plainte déposée par un ou des salariés victimes. Les lignes directrices de ces mesures seront établies par le MSTA. Dans les petites et moyennes entreprises, l'employeur devra mettre en place ces dispositifs à partir du $1^{\text {er }}$ avril 2023 et, en attendant cette date, est contraint d'engager tous les efforts adéquats pour leur future installation.

Ce nouvel article de la loi nº 24 du 5 mai 2019 oblige également l'employeur à faire suivre à son personnel une formation sur le harcèlement lié à des relations de pouvoir et/ou à prendre toutes les mesures de prévention nécessaires. En outre, l'employeur doit sensibiliser ses directeurs et cadres à ce type de harcèlement, ainsi qu'à la portée de leurs comportements et paroles envers leurs subordonnés.

En cas de différend avec sa hiérarchie, un salarié victime de ce type de harcèlement peut saisir le Directeur départemental du travail qui, à la suite d'une enquête, prodiguera à l'employeur des conseils, recommandations ou pistes de réflexion pour résoudre la 
situation. Dans l'hypothèse où l'employeur refuserait de suivre ces préconisations, le Directeur peut rendre son identité publique. Par ailleurs, ce différend peut également être confié à la Commission départementale de règlement amiable de conflit individuel de travail.

Un autre article figurant dans la loi n 24 de 2019 prohibe toute mesure discriminatoire susceptible d'être prise par l'employeur contre un salarié victime ayant porté plainte devant lui, ou devant la Commission susmentionnée, ou contre un salarié ayant témoigné en faveur de la victime. Enfin, la loi $n^{\circ} 24$ de 2019 a aussi apporté des modifications concernant le harcèlement sexuel lié à la maternité, qu'il s'agisse de comportements et paroles à l'égard d'une salariée enceinte partant en congé de maternité, d'un(e) salarié(e) partant en congé parental, ou d'un(e) salarié(e) reprenant son travail après ses congés. Les dispositions relatives à ces types de harcèlement ont été alignées sur celles concernant le harcèlement lié à des relations de pouvoir afin de renforcer la politique de la lutte contre toutes les formes de harcèlement.

\section{II - LE RENFORCEMENT DE LA PROTECTION DES ENFANTS CONTRE LES MALTRAITANCES ET VIOLENCES PARENTALES}

La protection des enfants contre les maltraitances et violences parentales, ainsi que leur prévention, sont des sujets qui attirent particulièrement l'attention de l'opinion publique, en raison notamment de la multiplication ces dernières années du nombre de tragédies causées par ces mauvaises conduites parentales. C'est principalement pour ce motif qu'a été adoptée la loi n 46 du 26 juin 2019 modifiant la loi sur l'action sociale en faveur des enfants et celle sur la prévention des maltraitances et violences à des enfants. Prenant en considération le fait que les violences parentales sont très souvent commises sous prétexte de corrections éducatives émanant de l'autorité parentale, la loi précise clairement qu'aucune violence physique ne peut être justifiée par la nécessité du droit de garde ou d'éducation prévus à l'article 820 du Code civil. En outre, cette loi déclare que les mesures inhérentes à l'exercice d'un droit disciplinaire parental instauré par le Code civil seront instaurées d'ici 2 ans, après la mise en vigueur de ladite loi et conformément aux propositions qui auront été formulées par le groupe de recherche nommé sur ce sujet au ministère de la justice.

Les Centres d'orientation de l'enfant, qui assurent la protection des enfants physiquement abusés par l'un ou ses parents, sont administrés par les Départements et recensent désormais un avocat-conseil pour les questions juridiques, un médecin et un infirmier de santé publique. La disponibilité de ces experts et le financement de leur rémunération restent néanmoins à l'étude. Les Départements doivent également fixer les nouvelles compétences territoriales de ces centres, en tenant compte notamment de l'évolution démographique afin de répondre aux critiques concernant leur sectorisation géographique inadaptée au développement territorial. La coordination entre ces centres d'une part, et ceux de l'orientation et du soutien des femmes victimes de violences domestiques d'autre part, sera organisée en considérant que la mère (l'épouse) et son enfant sont souvent victimes de violences émanant du (beau-)père (l'époux). L'article relatif à cette coordination a été inséré dans le projet de loi déposé par le Gouvernement à l'initiative de certains élus (en particulier des femmes) des partis de l'opposition. Enfin, le Gouvernement prendra les mesures nécessaires à l'augmentation de leur nombre et à l'amélioration de leur fonction. 


\section{III - LA GRATUITÉ DU SERVICE DE GARDE D'ENFANTS}

La politique familiale du Japon revêt une importance de plus en plus forte en raison notamment de la baisse de fécondité, du vieillissement rapide de la population, de la durabilité des activités économiques et du régime de la protection sociale. En témoigne I'adoption de la loi $n^{\circ} 7$ du 17 mai 2019 - modifiant la loi nº 65 du 22 août 2012 relative au soutien des enfants et des personnes qui s'en occupent - qui doit permettre de renforcer davantage la politique familiale.

Instaurée par la loi de 2012 susmentionnée, la "prestation de garde et d'éducation d'enfant dans une garderie ou une école maternelle agréées " est versée à un ou aux parents qui font garder leur(s) enfants dans ce type de structure. Sous le régime initial de la loi de 2012, les parents devaient verser à l'établissement agréé une participation aux frais de garde dont le montant est en partie fixé selon le revenu du foyer. Cette prestation ne couvrait pas donc la totalité des frais de garde engagés. Avec la loi de 2019, ce régime poursuit un objectif plus ambitieux: "la réduction de la charge financière d'une ou des personnes s'occupant d'un ou des enfants ». Depuis octobre 2019, cette participation est tout simplement supprimée pour tous les enfants âgés de 3 à 5 ans, ainsi que pour les enfants de 0 à 2 ans issus d'un foyer au faible revenu. Cette gratuité de garde d'enfant dans un établissement agréé devrait considérablement améliorer l'accès à ce service pour les jeunes couples avec enfants et travaillant tous les deux.

Par ailleurs, la loi de 2019 introduit une nouvelle prestation dite « prestation de recours à un établissement pour la garde et l'éducation d'enfant", afin d'augmenter le nombre d'établissements où les parents font garder leurs enfants. En effet, la gratuité évoquée ci-avant va encourager les jeunes couples à faire garder leur enfant alors que, parallèlement, les places dans les structures agréées sont insuffisantes, surtout dans les grandes villes. En créant cette nouvelle prestation, la loi de 2019 offre à ces couples la possibilité de recourir à des garderies d'enfants non agréées. Néanmoins, certains experts expriment déjà leur inquiétude quant à la qualité de la garde et à la sécurité des enfants dans ce type de garderies.

\section{IV - L'EMPLOI DES PERSONNES HANDICAPÉES DANS LE SECTEUR PUBLIC}

Les fraudes commises par les administrations en matière d'emploi public des personnes handicapées ${ }^{1}$ ont obligé le Gouvernement à déposer un projet de loi portant sur le renforcement de la politique et du contrôle de l'emploi des personnes handicapées dans le secteur public. Ce projet a été adopté et promulgué avec la loi n 36 du 14 mai 2019.

\section{V - LA DIFFÉRENCE INAPPROPRIÉE DE TRAITEMENT DU TRAVAILLEUR TITULAIRE D'UN CDD : ÉVOLUTION DE LA JURISPRUDENCE}

Deux arrêts de principe de la Cour suprême en 2018 portant sur la question de la différence du traitement entre salariés permanents à temps plein engagés sous CDI (salariés permanents) et ceux non permanents à temps plein engagés sous CDD (salarié CDD) sont déjà suivis par 6 arrêts des Cours d'appel.

1 Voir Revue de droit comparé du travail et de la sécurité sociale, n²019-1, p. 198. 
Selon ces 6 arrêts, sont considérés comme inappropriés le montant de certaines primes et indemnités plus élevé pour les salariés permanents que pour les salariés CDD, ou l'octroi de certains congés supplémentaires uniquement réservés aux salariés permanents. Cela concerne la prime d'assiduité, l'indemnité de logement, le congé payé complémentaire d'été et celui d'hiver, l'indemnité de travail en fin d'année et en début d'année suivante, le congé complémentaire pour blessure et maladie non professionnelles, le taux de majoration complémentaire des heures supplémentaires, la prime de retraite. En revanche, les juges ont rejeté le caractère inapproprié des montants différentiels entre les salariés permanents et les salariés CDD pour le salaire de base (même si le CDD de ces derniers a été renouvelé à plusieurs reprises ${ }^{2}$ ) et la prime de gratification d'été et d'hiver ${ }^{3}$.

Il convient néanmoins de souligner que, dans la majorité des affaires précitées, les salariés CDD plaignants pouvaient aisément être comparés aux permanents dans la mesure où les tâches assignées à ces deux catégories de salariés, par leur contrat de travail ou par l'instruction de la direction, étaient identiques. De plus, les conclusions adoptées par les juges dans ces affaires étant totalement casuistiques, il est difficile d'en tirer une orientation plus générale quant au caractère inapproprié d'un traitement différencié entre les salariés. Enfin, d'autres difficultés dans la pratique devraient survenir à compter du 1 er avril 2020, date à laquelle une réglementation plus stricte entrera en vigueur et impactera les salariés CDD et ceux à temps partiel sous CDI ou CDD. C'est alors la somme correspondant aux coûts de main-d'œuvre de tous les salariés - et non plus la répartition du revenu de l'entreprise entre l'employeur et les salariés - qui sera prise en considération dans les affaires de différence de traitement.

2 Cour d'appel de Tokyo, 20 février 2019. Mais dans l'affaire « L'Université de la santé du travail et de l'environnement »- Rodo Hanrei nº 1999 - 15 février 2019, la Cour d'appel d'Osaka avait jugé que le montant inférieur (par rapport à un salarié permanant) d'un salaire de base d'un salarié qui a une ancienneté de près de 30 ans par suite du renouvellement de son CDD, était inapproprié.

3 Cour d'appel de Tokyo, 13 décembre 2018; Cour d'appel d'Osaka, 24 janvier 2019. 